\title{
AARC Clinical Practice Guideline: Management of Pediatric Patients With Oxygen in the Acute Care Setting
}

\author{
Natalie Napolitano, Ariel Berlinski, Brian K Walsh, Emily Ginier, and Shawna L Strickland
}

\author{
Introduction \\ Committee Composition \\ Search Strategy \\ Study Selection \\ Development of Recommendations \\ Assessment and Recommendations \\ Oxygen Tents and Hoods Versus Low-Flow Oxygen Systems \\ High-Flow Oxygen Versus Low-Flow Oxygen \\ Humidification of Oxygen \\ Oxygenation Targets \\ Summary
}

\begin{abstract}
Oxygen therapy is one of the most important therapeutics offered in the clinical management of pediatric patients with cardiopulmonary disease. As the medical community seeks to ensure evidence-based management of clinical interventions, we conducted a systematic review with the goal of providing evidence-based clinical practice guidelines to answer questions surrounding the use of simple oxygen therapy to improve oxygenation, including a comparison of delivery devices, the efficacy of humidification, comparison of flows, and goals for use in children. Using a modification of the RAND/UCLA Appropriateness Method, we developed 4 recommendations to assist clinicians in the utilization of oxygen therapy in hospitalized children: (1) the use of an oxygen hood or tent in lieu of a low-flow oxygen device for consistent oxygen delivery is not recommended; (2) the use of high-flow nasal cannula therapy is safe and more effective than low-flow oxygen to treat infants with moderate to severe bronchiolitis; (3) the application of humidification with low-flow oxygen delivery is not recommended; (4) targeting $\mathrm{S}_{\mathrm{pO}_{2}} 90-97 \%$ for infants and children with bronchiolitis is recommended; however, no specific target can be recommended for pediatric patients with respiratory diseases outside of bronchiolitis, and establishing a patient/disease oxygen therapy target upon admission is considered best practice. Key words: oxygen; pediatric; child; infant. [Respir Care 2021;66(7):1214-1223. (C) 2021 Daedalus Enterprises]
\end{abstract}

\section{Introduction}

Oxygen is one of the most commonly delivered medications to children for the treatment of hypoxemia caused by many acute conditions such as lower respiratory tract infections, sepsis, or shock. Oxygen is widely considered to have many benefits and is applied liberally in clinical practice because it is inexpensive and because of the fear of longterm brain injury, including developmental and behavioral conditions, due to chronic and intermittent hypoxemia. ${ }^{1,2}$ Oxygen therapy does, however, have consequences of overuse and hyperoxia, such as oxygen toxicity and absorption atelectasis. ${ }^{3-5}$ The delivery of oxygen in pediatric patients requires appropriate selection of the delivery device, concentration, and flow for the most effective therapy to avoid both hypoxia and hyperoxia. ${ }^{6,7}$

The World Health Organization recommends oxygen delivery for $\mathrm{S}_{\mathrm{pO}_{2}}<90 \%$ for children with signs of respiratory 


\section{AarC Clinical Practice Guideline: Oxygen for Pediatric Patients}

distress. ${ }^{8}$ The American Heart Association's 2020 Pediatric Advance Life Support Guidelines state oxygen should be administered and weaned to achieve $\mathrm{S}_{\mathrm{pO}_{2}}$ between $94 \%$ and 99\%. ${ }^{9}$ The liberal use of oxygen in pediatric emergencies is not often questioned, but choosing parameters for treatment and establishing therapeutic goals remain highly variable. Therefore, we set out to develop evidence-based guidelines to help clinicians provide the best treatment of hypoxemia while limiting the adverse effects of oxygen therapy.

We conducted a systematic review of peer-reviewed literature to develop clinical practice guidelines to answer pressing questions in the management of pediatric patients with oxygen in the acute care setting. The following questions were developed to guide the systematic review:

1. In hospitalized pediatric patients, does the use of oxygen tents and hoods versus low-flow oxygen systems (nasal cannula, simple face mask) provide a more consistent oxygen delivery?

2. In hospitalized pediatric patients, does the use of high-flow oxygen systems versus low-flow oxygen systems increase the delivered fractional oxygen percentage or decrease escalation of therapy (noninvasive ventilation or intubation)?

3. In hospitalized pediatric patients, does adding humidification to supplemental oxygen as compared to no humidification improve comfort or reduce infection risk?

\footnotetext{
Ms Napolitano is affiliated with the Respiratory Therapy Department, Children's Hospital of Philadelphia, Philadelphia, Pennsylvania. Dr Berlinski is affiliated with the Division of Pediatric Pulmonary and Sleep Medicine, Department of Pediatrics, University of Arkansas for Medical Sciences, and Arkansas Children's Hospital, Little Rock, Arkansas. Dr Walsh is affiliated with the School of Health Sciences, Department of Allied Health Professions, Liberty University, Lynchburg, Virginia. Ms Ginier is affiliated with the Taubman Health Sciences Library, University of Michigan, Ann Arbor, Michigan. At the time of this research, Dr Strickland was affiliated with the American Association for Respiratory Care, Irving, Texas. She is now affiliated with the American Epilepsy Society, Chicago, Illinois.
}

Ms Napolitano has disclosed relationships with Drager, Philips/Respironics, Smiths Medical, and VERO-Biotech. Dr Berlinski has disclosed relationships with AbbVie, Allergan, Anthera, DCI, Cempra, Cystic Fibrosis Foundation, National Institute of Health, Mylan, Therapeutic Development Network, Trudell Medical International, Vertex, Vivus, and the International Pharmaceutical Aerosol Consortium on Regulation and Science. Dr Walsh has disclosed relationships with Vapotherm, Aerogen, Sentec, and VERO-Biotech. At the time of this work, Dr Strickland was an executive staff member of the American Association for Respiratory Care. Ms Ginier has no conflicts to disclose.

Supplementary material related to this paper is available at http://www. rcjournal.com.

Correspondence: Natalie Napolitano MPH RRT RRT-NPS FAARC, Respiratory Therapy Department, Children's Hospital of Philadelphia, 3401 Civic Center Blvd, 7NW148, Philadelphia, PA 19104. E-mail: napolitanon@chop.edu.

DOI: $10.4187 /$ respcare.09006
4. In hospitalized pediatric patients, does establishing disease-specific oxygenation targets reduce oxygen use, decrease the length of stay, or prevent escalation of therapy as compared to prescribed or no disease-specific oxygen targets?

\section{Committee Composition}

The committee for this endeavor was selected by the American Association for Respiratory Care (AARC) leadership on the basis of their known experience related to the topic, their interest in participating in the project, and their commitment to the process details. The committee first met face-to-face, where they were introduced to the process of developing clinical practice guidelines. At that time, the committee selected a chair and wrote a first draft of Patient, Intervention, Comparison, Outcome (PICO) questions. Subsequent meetings occurred as needed by conference call. Frequent e-mail communications occurred among committee members and AARC staff. The committee members received no remuneration for their participation in the process, though their expenses for the face-to-face meeting were reimbursed by the AARC.

\section{Search Strategy}

A literature search was conducted using the PubMed, CINAHL via EBSCOhost, and Scopus.com databases for studies on oxygen therapy in pediatric patients. The search strategies used a combination of relevant controlled vocabulary (ie, Medical Subject Headings and CINAHL Headings) and key word variations that were related to oxygen therapy, oxygenation techniques, pediatrics, and outcomes. The searches were limited to English-language studies about human populations published after 1986. The searches were also designed to filter out citations indexed as commentaries, editorials, interviews, news, or reviews. No date restrictions were applied to the searches, except for the cutoff to exclude studies published prior to 1987 . Refer to the online supplemental material for the complete search strategy executed in each database on January 10, 2020 (available at http://www. rcjournal.com). Cited reference searching was completed for all included studies as well as for studies that were topically relevant but excluded based on study design. Duplicate citations were identified and removed using the EndNote X8 citation management software (Clarivate, Philadelphia, Pennsylvania).

\section{Study Selection}

Two reviewers independently assessed study eligibility with the Covidence systematic review software (Covidence, Melbourne, Australia). Inclusion criteria used to assess 


\section{AarC Clinical Practice Guideline: Oxygen for Pediatric Patients}

eligibility were oxygen therapy; pediatric population, including neonates, infants, and children; and clinical outcomes. Exclusion criteria were not oxygen therapy; adult population; preterm newborn; no clinical outcomes relevant to oxygen therapy; not empirical research (eg, theory, opinion, or review articles); and published prior to 1987.

\section{Development of Recommendations}

A modification of the RAND/UCLA Appropriateness Method ${ }^{10}$ was used to combine the best available evidence with the collective experience of committee members. The literature was condensed into evidence tables according to PICO question (Table 1). Individual panel members were assigned the task of writing a systematic review of the topic, drafting $\geq 1$ recommendation, and suggesting the level of evidence supporting the recommendation:

Level A: Convincing scientific evidence based on randomized controlled trials of sufficient rigor.

Level B: Weaker scientific evidence based on lower levels of evidence such as cohort studies, retrospective studies, case-control studies, and cross-sectional studies.

Level C: Collective experience of the committee.

Committee members reviewed the first draft of evidence tables, systematic reviews, recommendations, and evidence levels. Each committee member rated each recommendation using a Likert scale from 1 to 9 , with 1 meaning expected harms greatly outweigh the expected benefits and 9 meaning expected benefits greatly outweigh the expected harms. The ratings were returned to the committee chair. The first ratings were done with no interaction among committee members. A conference call was convened, during which the individual committee ratings were discussed. Particular attention was given to any outlier scores and the justification. Recommendations and evidence levels were revised with input from the committee members. After discussing each PICO question, committee members re-rated each recommendation. The final median and range of committee members' scores are reported (Table 2). Strong agreement required that all committee members rank the recommendation as 7 or higher, whereas weak agreement meant that $\geq 1$ committee member ranked the recommendation below 7, but the median vote was at least 7 . For recommendations with weak agreement, the percentage of committee members who rated at 7 or above was calculated and reported after each weak recommendation. Figure 1 illustrates the process flow used by the panel to rate the appropriateness and quality of the literature selected through the search process.

Drafts of the report were distributed among committee members in several iterations. When all committee members were satisfied, the document was submitted for publication. The report was subjected to peer review before the final publication.

\section{Assessment and Recommendations}

The search strategies retrieved a total of 3,312 articles. The team also identified 1,413 articles through the cited references of articles assessed for full-text eligibility and 1 relevant article through other methods. After removal of duplicates, 4,116 articles remained for screening. In the title and abstract screening phase, 3,950 articles were excluded. Of the remaining 166 articles, 153 were excluded following full-text review against the inclusion and exclusion criteria (Fig. 2). Search results were assessed according to PRISMA guidelines (www.prisma-statement.org. Accessed May 10, 2021).

\section{Oxygen Tents and Hoods Versus Low-Flow Oxygen Systems}

Oxygen can be delivered to hospitalized pediatric patients in a variety of ways. Most pediatric patients receive supplemental oxygen via low-flow nasal cannula devices or via simple face mask. The inspired oxygen provided to the patient is dependent upon gas flow, the patient's inspiratory demand, and the diameter of the nares. A simple face mask may deliver more inspired oxygen to the pediatric patient, but the mask is harder to secure to the patient's face, and it is imperative that a minimum flow is used to flush exhaled carbon dioxide. ${ }^{6}$

Historically, hospitalized pediatric patients received oxygen via humidified tent or hood. The oxygen tent, also called a mist tent or croup tent, consists of clear plastic sheeting that hangs over the patient's hospital bed. The tent allows for an input of cool, humidified oxygen from high-pressure gas sources. Popular for its ability to allow the child movement within the tent in the hospital bed, there are many disadvantages to this method of oxygen delivery: the maximum $\mathrm{F}_{\mathrm{IO}_{2}}$ achieved is around 0.4 and is dependent upon how well the tent is secured around the patient and bed; children could bring spark-emitting toys within the tent environment, which could support combustion; and the plastic could be a source of suffocation. In addition, these large tents are difficult to set up and maintain, as microbial contamination is likely. ${ }^{6,23-25}$

Oxygen hoods consist of a collapsible plastic cube that surrounds the head of the infant. Both devices function in the same way and may theoretically deliver an $\mathrm{F}_{\mathrm{IO}_{2}}$ up to 1.0 with appropriate gas flow, and both devices require a minimum gas flow to flush exhaled carbon dioxide from the device. Similar to the tent, the efficacy of oxygen delivery with this device is dependent upon the seal of the device around the patient's head. The larger the unsealed area, the less likely the target will be reached. ${ }^{23}$ 


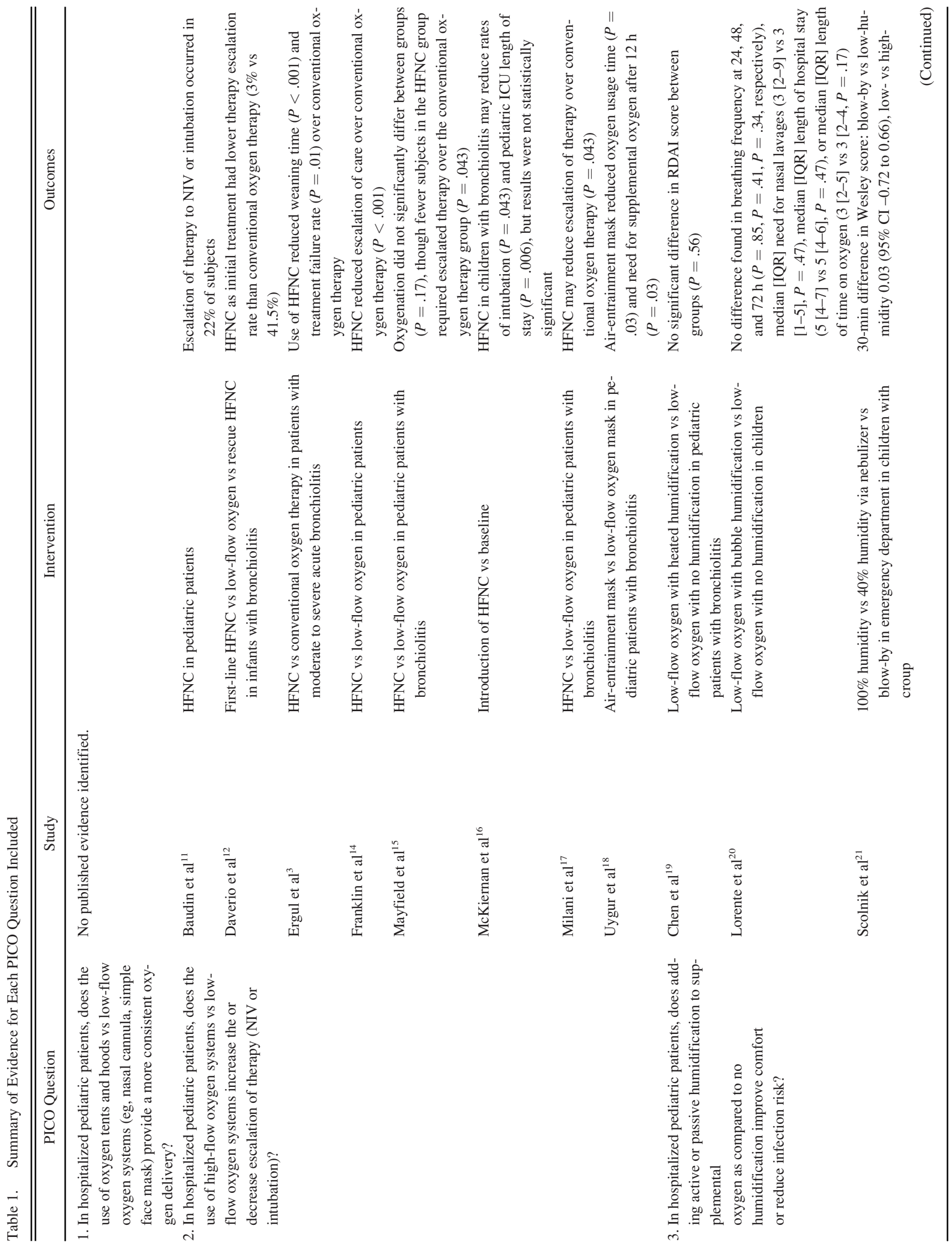




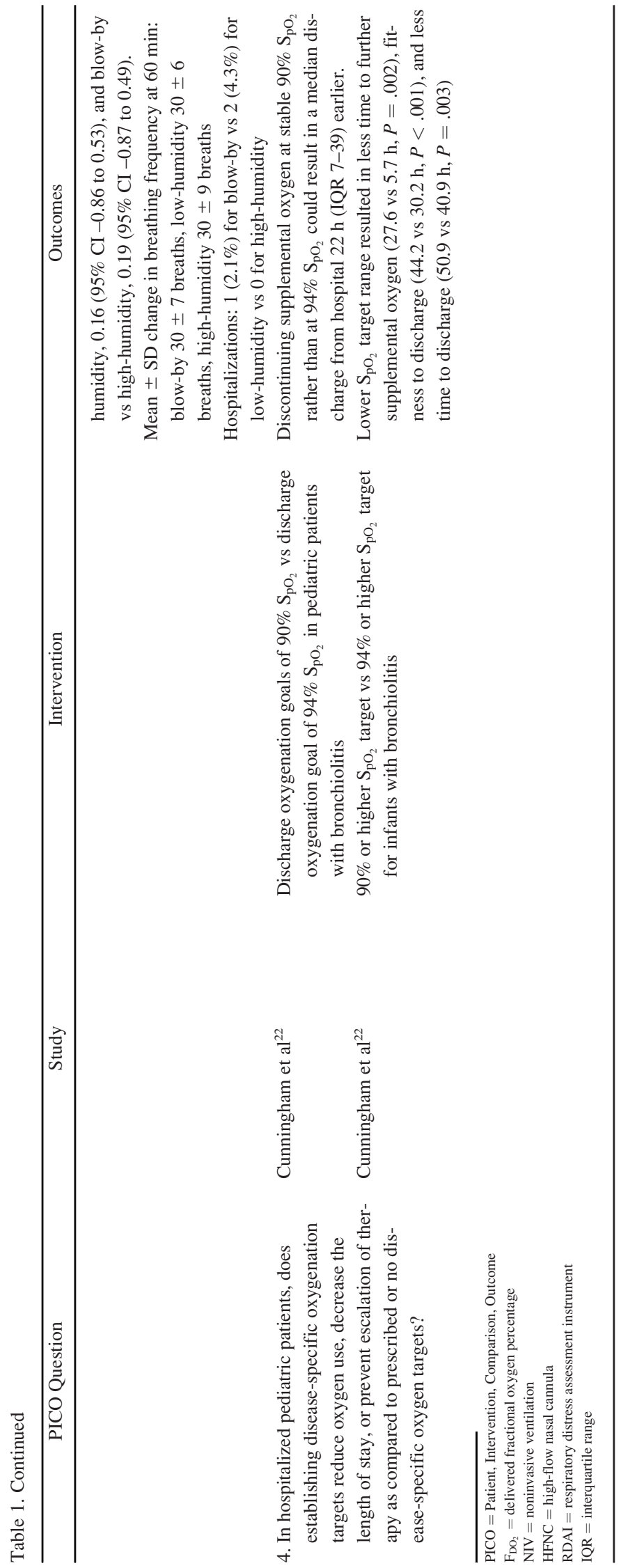

Mist tents, smaller oxygen tents, and oxygen hoods are all subject to lower delivered $\mathrm{F}_{\mathrm{IO}_{2}}$ due to structural issues with creating a sealed environment. Though there are limitations to achieving consistent oxygen delivery with low-flow devices such as the nasal cannula and simple face mask, there is a lack of evidence comparing the consistency of oxygen delivery between the oxygen hood, oxygen tent, and low-flow devices. As such, the committee does not recommend using an oxygen hood or tent in lieu of a low-flow oxygen device for consistent oxygen delivery (Evidence level C, median appropriateness score 8).

\section{High-Flow Oxygen Versus Low-Flow Oxygen}

The use of high-flow nasal cannula (HFNC) systems to provide respiratory support to children has increased over the past decade. These systems have the potential to provide washout of the nasopharyngeal dead space; decrease inspiratory resistance; improve airways conductance and pulmonary compliance; and decrease metabolic work by providing heated and humidified gas. ${ }^{26}$ Finally, the generation of positive pressure is a more controversial mechanism. Although these systems were initially used in pediatric ICU settings, they have transitioned to the acute care setting. Few studies have compared the use of low-flow and HFNC systems for the treatment of respiratory conditions in infants and children. One of the challenges in analyzing the published data is the variable definition of HFNC among publications. Another limitation is that, with few exceptions, most studies are retrospective and have a small sample size. Another confounder is that all of the reported studies included infants diagnosed with either moderate or severe bronchiolitis. Therefore, it is not completely clear whether the benefits of HFNC arise from improved oxygenation only or by also providing more robust ventilatory support.

Franklin et $\mathrm{al}^{14}$ reported a multi-center, randomized controlled clinical trial comparing low-flow oxygen (up to $2 \mathrm{~L} / \mathrm{min}$ ) and HFNC ( $2 \mathrm{~L} / \mathrm{kg} / \mathrm{min}$ ) to treat 1,472 infants $<12$ months old admitted to the pediatric ward with a diagnosis of bronchiolitis who needed supplemental oxygen therapy. This is the largest reported study to date comparing low-flow and HFNC therapies. The primary outcome was a need for escalation of care defined as the presence of 3 of 4 criteria (ie, unchanged or elevated heart rate and breathing frequency, hypoxemia at $2 \mathrm{~L} / \mathrm{min}$ or $40 \%$ with HFNC, triggering of early warning tool). Subjects assigned to treatment with HFNC had a lower rate of escalation of care than those on the low-flow oxygen arm (12\% vs $23 \%) .{ }^{14} \mathrm{~A}$ large percentage of subjects (61\%) who 


\section{AarC Clinical Practice Guideline: Oxygen for Pediatric Patients}

Table 2. Summary of Recommendations for Each PICO Question

1. In hospitalized pediatric patients, does the use of oxygen tents and hoods vs low-flow oxygen systems (eg, nasal cannula, simple face mask) provide a more consistent oxygen delivery?

The committee does not recommend using an oxygen hood or tent in lieu of a low-flow oxygen device for consistent oxygen delivery (Evidence level C, median appropriateness score 8).

2. In hospitalized pediatric patients, does the use of high-flow oxygen systems vs low-flow oxygen systems increase the $\mathrm{F}_{\mathrm{DO}_{2}}$ or decrease escalation of therapy (noninvasive ventilation or intubation)?

The use of HFNC appears to be safe and more effective than low-flow oxygen to treat infants with moderate to severe bronchiolitis in the pediatric ward and pediatric ICU settings (Evidence level B, median appropriateness score 7.5).

3. In hospitalized pediatric patients, does adding active or passive humidification to supplemental oxygen as compared to no humidification improve comfort or reduce infection risk?

Low level of evidence does not support the use of heated or non-heated humidification with low-flow oxygen delivery (Evidence level B, median appropriateness score 8.25).

4. In hospitalized pediatric patients, does establishing disease-specific oxygenation targets reduce oxygen use, decrease length of stay, or prevent escalation of therapy as compared to prescribed or no disease-specific oxygen targets?

In hospitalized pediatric patients suffering from bronchiolitis, evidence supports an oxygenation target of $90 \%$ or greater (Evidence level C, median appropriateness score 7).

In hospitalized pediatric patients suffering from respiratory diseases outside of bronchiolitis, establishing a patient/disease oxygen therapy target upon admission is best practice, but a specific target cannot be recommended (Evidence level C, median appropriateness score 7).

$\overline{\mathrm{PICO}}=$ Patient, Intervention, Comparison, Outcome

$\mathrm{F}_{\mathrm{DO}_{2}}=$ delivered fractional oxygen percentage

$\mathrm{HFNC}=$ high-flow nasal cannula

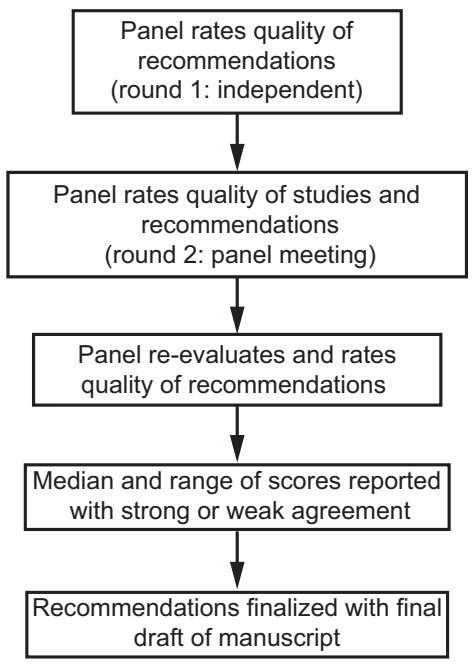

Fig. 1. A flow diagram outlining the process used by the committee to appraise the literature.

failed low-flow oxygen responded to HFNC. Hospital length of stay, duration of oxygen therapy, and incidence of pneumothorax $(0.1 \%)$ were similar between groups. ${ }^{14}$

Ergul et $\mathrm{al}^{13}$ reported a randomized trial comparing an oxygen mask ( $n=30$ subjects) to HFNC ( $n=30$ subjects) in the treatment of infants 1-24 months old admitted to a pediatric ICU with a diagnosis of moderate to severe bronchiolitis. Subjects in the HFNC group required almost 50\% less time on supplemental oxygen support, did not experience treatment failure (ie, escalation of care, lack of improvement in heart rate and breathing frequency, and persistent hypoxemia), and had shorter pediatric ICU and hospital stays than subjects in oxygen mask group. ${ }^{13}$ Uygur et $\mathrm{al}^{18}$ reported a prospective clinical trial in children 3-36 months old admitted to a pediatric ward with hypoxemic acute lower respiratory infection. The subjects were randomized to simple mask ( $n=32$ subjects) or air entrainment mask ( $n=33$ subjects). Although both groups improved, those in the air-entrainment mask group had a greater reduction in breathing frequency at $24 \mathrm{~h}$ than those in the simple mask group. In addition, subjects treated with the air entrainment mask had a 50\% reduction in the duration of time requiring supplemental oxygen. ${ }^{18}$

In a nonformally randomized clinical trial, Milani et $\mathrm{al}^{17}$ compared the use of HFNC (where $n=20$ subjects) versus low-flow oxygen (no upper limit defined; $n=20$ subjects) to treat infants $<12$ months old admitted to a pediatric ward with a diagnosis of moderate to severe bronchiolitis who needed supplemental oxygen therapy. Those receiving HFNC experienced a faster reduction in breathing frequency, regained their ability to feed faster, and required less time on supplemental oxygen and shorter hospitalization than those receiving low-flow oxygen. None of the subjects required pediatric ICU admission. ${ }^{17}$

Mayfield et $\mathrm{al}^{15}$ conducted a pilot study to compare use of HFNC $(2 \mathrm{~L} / \mathrm{kg} / \mathrm{min})(n=61$ subjects) with low-flow oxygen $(n=31$ subjects $)$ in infants $<12$ months old admitted to a pediatric ward with a diagnosis of bronchiolitis who needed supplemental oxygen therapy. Subjects were enrolled in this HFNC pilot study, and the low-flow group was retrospectively identified. Subjects in the HFNC group were less likely to require transfer to pediatric ICU (13\% vs 


\section{AarC Clinical Practice Guideline: Oxygen for Pediatric Patients}

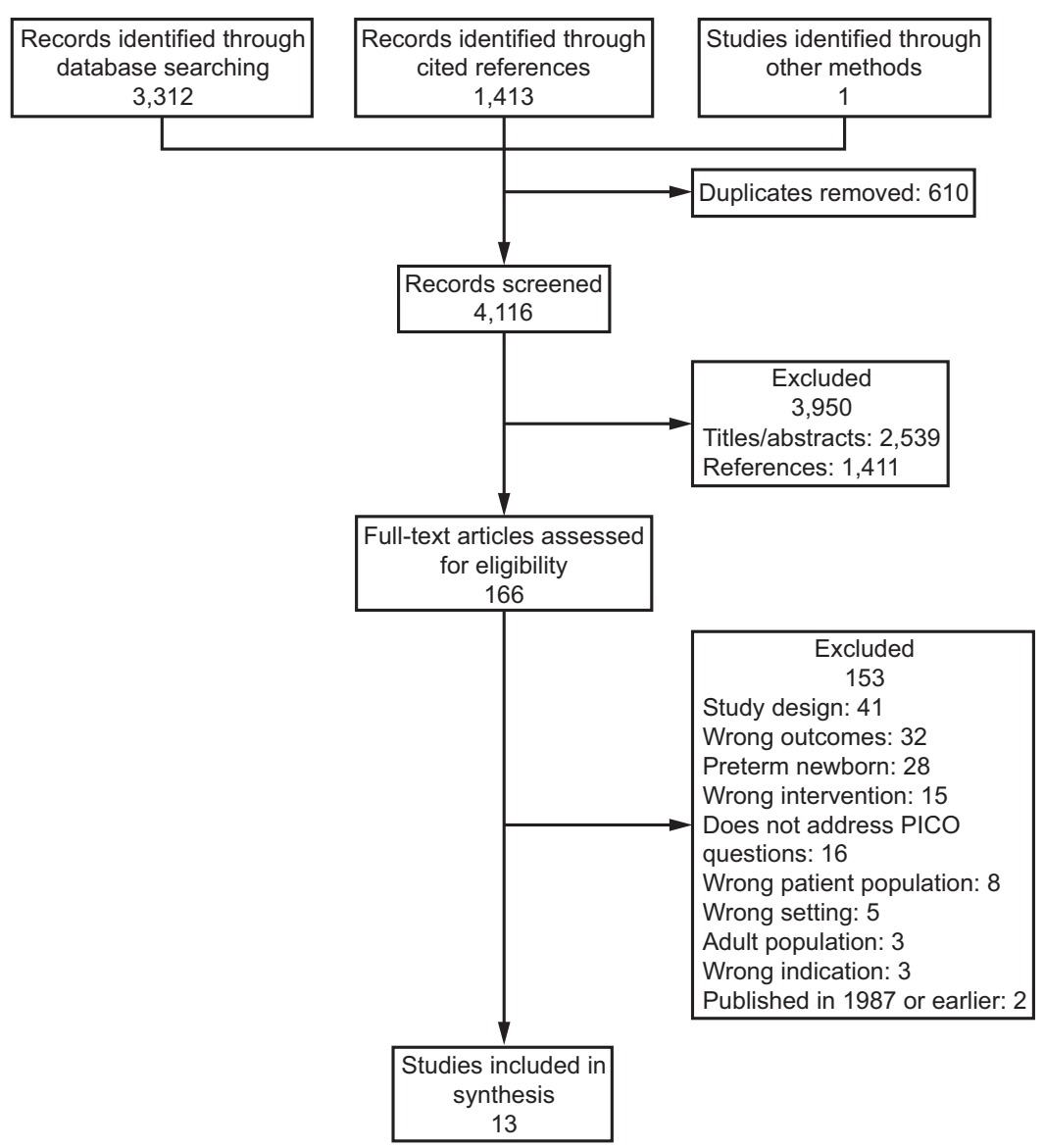

Fig. 2. Flow chart.

$30 \%) .{ }^{15}$ The authors reported that a lack of decrease in breathing frequency and heart rate at the first hour of HFNC was associated with treatment failure. Length of hospital stay was similar between groups. ${ }^{15}$

Daverio et al ${ }^{12}$ retrospectively reviewed their 5-y experience using a 2-tiered HFNC (2 L/kg/min) protocol in 211 infants $<12$ months old who were admitted to the pediatric ward of a tertiary care hospital with a diagnosis of bronchiolitis. The authors used a standardized score to decide the type of respiratory support (low-flow vs HFNC). Most subjects $(83 \%)$ were started on low-flow oxygen $(<2$ $\mathrm{L} / \mathrm{min}$ ), but $41 \%$ of them required the use of rescue HFNC. ${ }^{12}$ These subjects had shorter duration of illness, lower body weight $\mathrm{Z}$-score, and lower $\mathrm{S}_{\mathrm{pO}_{2}}$ on arrival; these subjects also received more epinephrine treatments and were more likely to have cardiac comorbidity than those who did not need escalation. Air leaks were reported in $1.9 \%$ of subjects receiving HFNC. Transfer to pediatric ICU was low for both groups (5\% and 3\% for low-flow and HFNC groups, respectively). ${ }^{12}$

McKiernan et $\mathrm{al}^{16}$ retrospectively reviewed intubation rates in infants $<24$ months of age admitted to a pediatric ICU with a diagnosis of bronchiolitis. The authors compared 2 treatment periods: before ( $n=57$ subjects) and after ( $n=58$ subjects) use of HFNC for treatment of bronchiolitis. They reported a $68 \%$ reduction in intubation rate after HFNC, and the reduction remained statistically significant after adjusting for age, weight, and respiratory syncytial virus status. ${ }^{16}$ Similar to Mayfield et al, ${ }^{15}$ McKiernan et $\mathrm{al}^{16}$ reported that infants who did not reduce their breathing frequency after starting HFNC therapy were more likely to be intubated.

Baudin et $\mathrm{al}^{11}$ retrospectively reviewed the safety of using HFNC in pediatric patients admitted to a pediatric ICU in a tertiary care hospital. The authors reviewed 177 episodes corresponding to 145 subjects (median [interquartile range] 8 [2-28] months) over a 1-y data period. ${ }^{11}$ The system was used for de-escalation of support (36\% and $18 \%$ after extubation and NIV, respectively) and as primary support (31\%). ${ }^{11}$ The maximum flow used was $<2$ $\mathrm{L} / \mathrm{kg} / \mathrm{min}$, and a $22 \%$ failure rate was reported. The authors reported a low rate of complications $(1.0 \%$ and $0.6 \%$ for pneumothorax and epistaxis, respectively). ${ }^{11}$

In summary, the use of HFNC ( $\leq 2 \mathrm{~L} / \mathrm{kg} / \mathrm{min})$ appears to be safe and more effective than low-flow oxygen $(<2$ $\mathrm{L} / \mathrm{min}$ ) to treat infants with moderate to severe bronchiolitis 


\section{AarC Clinical Practice Guideline: Oxygen for Pediatric Patients}

in the pediatric ward and the pediatric ICU settings (Evidence level B, median appropriateness score 7.5).

\section{Humidification of Oxygen}

The use of humidification for low-flow oxygen therapy is a common practice with little evidence to support its use or to indicate that it is harmful. Complications of dry oxygen delivery have been linked to the use of high-flow oxygen delivery and are perceived to be present with lower severity with low-flow oxygen delivery. There are 2 options for humidification of low flow oxygen. The first and most common is unheated humidification, also referred to as bubble or pass-over, and the second is heated and humidified humidification via an active humidification device.

The evidence to support the use of humidification of any kind versus dry delivery of low-flow oxygen delivery to impact comfort or infection risk is scant. Three studies to date have investigated this comparison. Scolnik et $\mathrm{al}^{21}$ concentrated on the croup population in the emergency department and performed a single-blinded, randomized controlled trial to deliver 3 levels of humidity for $30 \mathrm{~min}$ in the emergency department. The 3 groups were blow-by therapy, controlled $40 \%$ humidity, and controlled $100 \%$ humidity, all delivered with $40 \%$ oxygen, and pre- and postassessment was conducted with the Westley Croup Score. The results showed that there was no significant difference in the Westley score or any of the secondary outcomes of vital signs and hospitalization rate with either level of humidity, which did not support the use of humidification in the emergency department for the treatment of croup. ${ }^{21}$ The HHOT AIR study by Chen et $\mathrm{al}^{19}$ was a prospective, randomized pilot study comparing dry versus heated and humidified low-flow oxygen defined as $<4 \mathrm{~L} / \mathrm{min}$ in children $<24$ months of age with mild to moderate bronchiolitis admitted to the general pediatric ward. Patients who required oxygen therapy were randomized to the control or to heat and humidification for oxygen delivery. The respiratory distress assessment instrument (RDAI) was used to score comfort and distress to compare for each group. Although the investigators saw a quicker change in the RDAI over time ( $1 \mathrm{~h} \mathrm{vs} 12 \mathrm{~h}$ ), there was not a significant difference in the score when comparing the 2 groups at the same timeframes. Although there was a trend in a quicker improvement of RDAI over time with the heated and humidified oxygen group, this did not show a significant difference in length of stay or vital signs. This may be a result of small sample size; however, these findings do not support the use of heated and humidified oxygen over dry gas for the treatment of bronchiolitis. Lorente Sánchez et al also investigated the use of humidification of low-flow oxygen therapy in children with mild to moderate bronchiolitis admitted to the general pediatric ward in a pre-post interventional trial. ${ }^{20}$ The authors used unheated humidification and compared the number of nasal lavages preand post-universal use of a bubbler humidifier and changes in bronchiolitis score, vital signs, and hospital length of stay. ${ }^{20}$ There was not a statistical difference in any outcome measure, indicating that the use of unheated humidification was not beneficial.

In summary, low level of evidence does not support the use of heated or unheated humidification with low-flow oxygen delivery (Evidence level B, median appropriateness score 8.25). More robust research is needed to make a clear determination.

\section{Oxygenation Targets}

Normoxia, or a pulse oximetry reading of $94 \%$ or higher, is often the recommended target for oxygen therapy in children. There are several clinical practice guidelines that recommend pulse oximetry targets by disease, yet practice and even recommendations vary between national guidelines. Current oximetry target recommendations for children with pneumonia are $>92 \%$ and $>90 \%$ for asthma if using the 2007 NHLBI National Asthma Education and Prevention Program, or 94-98\% if using the revised 2019 British guideline on the management of asthma. ${ }^{27-29}$ We identified only one category of respiratory disease in which there was enough evidence of specific oxygen targets in the pediatric population. This respiratory disease was acute lower respiratory tract infection, often referred to as bronchiolitis.

Lower respiratory tract infections account for about 128,000 hospitalizations of children $<2$ y old each year in the United States at an estimated cost of $\$ 1.73$ billion, which is an estimated $30 \%$ increase over the 9 y studied. ${ }^{30}$ While trends in hospitalization for lower respiratory tract infections have decreased from 17.9 to 14.9 per 1,000 persons from 2000 to 2009, there was a $34 \%$ increase in admissions of children with high-risk medical conditions and a $21 \%$ increase in use of mechanical ventilation. ${ }^{30}$ The American Academy of Pediatrics and the World Health Organization both recommend permissive hypoxemia of an oxygen saturation of $90 \%$ for children with lower respiratory tract infections, largely on the basis of expert opinion and consideration of resource-limited areas as there was not a high level of evidence presented in their publications. ${ }^{31,32}$

Since the publication of those national clinical practice guidelines on the management of bronchiolitis, there has been one double-blind, randomized, equivalence trial using 2 oximetry targets $(<90 \%$ modified vs $<94 \%$ standard $)$ for oxygen therapy. ${ }^{22}$ Cunningham et $\mathrm{al}^{22}$ reported that the modified target of $\geq 90 \%$ is as safe and clinically effective as the standard practice of $\geq 94 \%$. Additionally, they observed a significantly lower time to fit to discharge (44.2 vs $30.2 \mathrm{~h}, P<.001$ ), time to actual discharge (50.9 vs 40.9 h, $P=.003$ ), and time to no further supplemental oxygen 


\section{AarC Clinical Practice Guideline: Oxygen for Pediatric Patients}

(27.6 vs $5.7 \mathrm{~h}, P=.002$ ) in the modified oxygen saturation group. $^{22}$ This was all accomplished without any differences in adverse events and safety outcomes.

In hospitalized pediatric patients with bronchiolitis, evidence supports an oxygenation target of $\geq 90 \%$ (Evidence level C, median appropriateness score 7). However, in hospitalized pediatric patients with respiratory diseases other than bronchiolitis, establishing a patient/disease oxygen therapy target upon admission is best practice, but a specific target cannot be recommended (Evidence level C, median appropriateness score 7).

\section{Summary}

The results of this systematic review are summarized in Table 1. Disappointingly, the evidence available to answer the PICO questions is minimal. The recommendations in Table 2 are based on low levels of evidence and strongly reflect committee experience. The PICO questions were developed from the aspects of oxygen use that were perceived to have the highest variability in clinical practice and the greatest chance of reducing the efficiencies in care of pediatric patients. The result of this evidence-based review have identified more needs for future research than evidence-based recommendations for clinical practice. The lack of evidence to support the consistency of oxygen delivery via oxygen tents and hoods over the use of low-flow oxygen devices is overwhelmed by the potential risks.

The use of HFNC ( $\leq 2 \mathrm{~L} / \mathrm{kg} / \mathrm{min})$ appears to be safe and more effective than low-flow oxygen $(<2 \mathrm{~L} / \mathrm{min})$ to treat infants with moderate to severe bronchiolitis in the pediatric ward and pediatric ICU settings. Practitioners need to use appropriately sized cannulas that block around $50 \%$ of naris openings. Larger sizes increase the risk of inadvertently creating PEEP and pressure ulcers. Also, the cannulas should not exceed the manufacturers' recommended maximum flow. Exceeding these flows results in the buildup of back pressure. It is important to understand the role of HFNC in pediatric oxygenation and to be mindful of its limitations. Protocols that include indications, contraindications, escalation, and de-escalation of care, scheduled monitoring of occurrence of pressure ulcers, recommendations for obtaining a blood gas, and transfer to pediatric ICU if used in the ward should be used. Monitoring adverse events that occur during the use of HFNC should be included in the protocols. In addition, when HFNC is used in pediatric wards, patients should be monitored with continuous pulse oximetry, and staff need to be appropriately trained to use the devices and to recognize complications. Although studies done in conditions other than bronchiolitis are lacking, these systems are used in clinical practice. This is an area that needs further study. In the meantime, if used, careful monitoring is of the utmost importance.
There are few investigations into the benefit of adding humidification (heated or unheated) for low-flow oxygen delivery. None of the studies identified showed a difference in the defined outcomes. From these results, the committee cannot recommend the routine use of humidification for low-flow oxygen delivery. There is a need for research into the shortand long-term outcomes for each type of humidification.

Titration of oxygen to a therapeutic goal in the treatment of hypoxemia would appear to be a best practice within many of the disease-specific clinical practice guidelines today, but, apart from bronchiolitis, this practice has not been evaluated scientifically. In hospitalized patients with bronchiolitis, targeting a lower-than-normal oxygen saturation results in less time receiving oxygen therapy and earlier discharge. In hospitalized pediatric patients with bronchiolitis, evidence supports an oxygenation saturation target of $90-98 \%$. While expert clinicians recommend therapeutic oxygenation targets for respiratory diseases, we could find little evidence that these targets are correct. In hospitalized pediatric patients with respiratory diseases other than bronchiolitis, establishing a patient/disease oxygen therapy target upon admission is best practice, but a specific target cannot be recommended.

\section{ACKNOWLEDGMENTS}

We thank Ms Melissa Brown for her contribution to the development of the research questions, literature review, and data extraction.

\section{REFERENCES}

1. Subhi R, Adamson M, Campbell H, Weber M, Smith K, Duke T, Hypoxaemia in Developing Countries Study Group. The prevalence of hypoxaemia among ill children in developing countries: a systematic review. Lancet Infect Dis 2009;9(4):219-227.

2. Bass JL, Corwin M, Gozal D, Moore C, Nishida H, Parker S, et al. The effect of chronic intermittent hypoxemia on cognition in childhood: a review of the evidence. Pediatrics 2004;114(3):805-816.

3. Kacmarek RM, Stoller JK, Heur AJ. Egan's fundamentals of respiratory care, 10th edition. St Louis: Mosby Elsevier; 2013:321, 322, 914.

4. Nunn JF. Conscious volunteers developed hypoxemia and pulmonary collapse when breathing air and oxygen at reduced lung volumes. Anesthesiology 2003;98(1):258-259.

5. Saugstad OD. Bronchopulmonary dysplasia-oxidative stress and antioxidants. Semin Neonatol 2003;8(1):39-49.

6. Walsh BK, Smallwood CD. Pediatric oxygen: a review and update. Respir Care 2017;62(6):645-661.

7. Rojas-Reyes MX, Rugeles CG, Charry-Anzola LP. Oxygen therapy for lower respiratory tract infections in children between 3 months and 15 years of age. Cochrane Database Syst Rev 2014;2014(12):CD005975.

8. World Health Organization. Guideline: updates on paediatric emergency triage, assessment and treatment. Geneva: World Health Organization; 2016.

9. Topjian AA, Raymond TT, Atkins D, Chan M, Duff JP, Joyner BL, et al. Part 4: pediatric basic and advanced life support: 2020 American Heart Association guidelines for cardiopulmonary resuscitation and emergency cardiovascular care. Circulation 2020;142(16 suppl 2):S469-S523.

10. Fitch K, Bernstein SJ, Aguilar MD, Burnand B, LaCalle JR, Lazaro P, et al. The RAND/UCLA Appropriateness Method User's Manual. Santa Monica, CA: RAND; 2001. 


\section{Aarc Clinical Practice Guideline: Oxygen for Pediatric Patients}

11. Baudin F, Gagnon S, Crulli B, Proulx F, Jouvet P, Emeriaud G. Modalities and complications associated with the use of high-flow nasal cannula: experience in a pediatric ICU. Respir Care 2016;61 (10):1305-1310.

12. Daverio M, Da Dalt L, Panozzo M, Frigo AC, Bressan S. A two-tiered high-flow nasal cannula approach to bronchiolitis was associated with low admission rate to intensive care and no adverse outcomes. Acta Paediatr 2019;108(11):2056-2062

13. Ergul AB, Calıskan E, Samsa H, Gokcek I, Kaya A, Zararsiz GE, Torun YA. Using a high-flow nasal cannula provides superior results to OxyMask delivery in moderate to severe bronchiolitis: a randomized controlled study. Eur J Pediatr 2018;177(8):1299-1307.

14. Franklin D, Babl FE, Schlapbach LJ, Oakley E, Craig S, Neutze J, et al. A randomized trial of high-flow oxygen therapy in infants with bronchiolitis. N Engl J Med 2018;378(12):1121-1131.

15. Mayfield S, Bogossian F, O'Malley L, Schibler A. High-flow nasal cannula oxygen therapy for infants with bronchiolitis: pilot study. J Paediatr Child Health 2014;50(5):373-378.

16. McKiernan C, Chua LC, Visintainer PF, Allen H. High flow nasal cannulae therapy in infants with bronchiolitis. J Pediatr 2010;156(4):634-638.

17. Milani GP, Plebani AM, Arturi E, Brusa D, Esposito S, Dell'Era L, et al. Using a high-flow nasal cannula provided superior results to low-flow oxygen delivery in moderate to severe bronchiolitis. Acta Paediatr 2016;105(8):e368-e372.

18. Uygur P, Oktem S, Boran P, Tutar E, Tokuc G. Low- versus high-flow oxygen delivery systems in children with lower respiratory infection. Pediatr Int 2016;58(1):49-52.

19. Chen DY, Zee ED, Gildengorin G, Fong EW. A pilot study of heated and humidified low flow oxygen therapy: an assessment in infants with mild and moderate bronchiolitis (HHOT AIR study). Pediatr Pulmonol 2019;54(5):620-627.

20. Lorente Sánchez S, Gimeno R, Losilla JM, Garzón S, Vives J. Benefits of the humidified low-flow oxygen therapy in infants with mild-moderate bronchiolitis. J Clin Nurs 2018;27(5-6):1125-1133.
21. Scolnik D, Coates AL, Stephens D, Da Silva Z, Lavine E, Schuh S. Controlled delivery of high vs low humidity vs mist therapy for croup in emergency departments: a randomized controlled trial. JAMA 2006;295(11):1274-1280.

22. Cunningham S, Rodriguez A, Adams T, Boyd KA, Butcher I, Enderby B, et al. Oxygen saturation targets in infants with bronchiolitis (BIDS): a double-blind, randomised, equivalence trial. Lancet 2015;386(9998):1041-1048.

23. Walsh BK, Brooks TM, Grenier BM. Oxygen therapy in the neonatal care environment. Respir Care 2009;54(9):1193-1202.

24. Rubin BK, Haynes JM. Myths, misunderstandings, and dogma in respiratory care. Respir Care 2012;57(8):1314-1324.

25. Volsko TA. Cystic fibrosis and the respiratory therapist: a 50-year perspective. Respir Care 2009;54(5):587-594.

26. Dysart K, Miller TL, Wolfson MR, Shaffer TH. Research in high flow therapy: mechanisms of action. Respir Med 2009;103(10):1400-1405.

27. Harris M, Clark J, Coote N, Fletcher P, Harnden A, McKean M, et al. British Thoracic Society guidelines for the management of community acquired pneumonia in children: update 2011. Thorax 2011;66(Suppl 2):ii1-ii23.

28. National Asthma Education and Prevention Program. Expert panel report 3 (EPR-3): guidelines for the diagnosis and management of asthma: summary report. J Allergy Clin Immunol 2007;120(5 Suppl):S94-S138.

29. British Thoracic Society Scottish Intercollegiate Guidelines Network. SIGN 158 British guideline on the management of asthma. London: British Thoracic Society; 2019:172.

30. Hasegawa K, Tsugawa Y, Brown DF, Mansbach JM, Camargo CA, Jr. Trends in bronchiolitis hospitalizations in the United States, 2000 2009. Pediatrics 2013;132(1):28-36.

31. Ralston SL, Lieberthal AS, Meissner HC, Alverson BK, Baley JE, Gadomski AM, et al. Clinical practice guideline: the diagnosis, management, and prevention of bronchiolitis. Pediatrics 2014;134(5):e1474-e1502.

32. World Health Organization. WHO Pocket book of hospital care for children. Geneva: WHO Publications; 2013:412. 